\title{
¿CÓMO ESCRIBEN LOS APRENDICES HISPANOHABLANTES DE NIVEL INTERMEDIO EN RUSO COMO LE? UN ESTUDIO SOBRE LA INFLUENCIA FONOLÓGICA DE L1 EN LE
}

\author{
HOW DO SPANISH-SPEAKING LEARNERS WITH INTERMEDIATE LEVEL \\ WRITE IN RUSSIAN AS FL? A STUDY ON THE PHONOLOGICAL EFFECT \\ OF L1 ON FL
}

\author{
Anastasila Ogneva \\ Universidad de A Coruña, España \\ anastasiia.ogneva@udc.es \\ https://orcid.org/0000-0003-0237-7146
}

\begin{abstract}
Resumen
En este trabajo se presentan los resultados del análisis de un corpus compuesto de 30 producciones escritas realizadas por aprendices adultos hispanohablantes de ruso como LE. El objetivo del trabajo es identificar los errores ortográficos y sus causas en el nivel B2, así como comparar el número total de errores ortográficos que comenten dos grupos de aprendices de diferentes niveles - A2 y B2. El análisis ha reflejado que los errores más frecuentes son los de sustitución de las consonantes $\sigma-\beta[\mathrm{b}]-[\mathrm{v}]$, de las vocales $u$-bl [i]-[i] y del signo blando. La causa de los errores se encuentra en las diferencias existen-
\end{abstract}

\begin{abstract}
This paper reports on the results of a corpus analysis of 30 written works produced by Spanish-speaking learners of Russian as a foreign language (FL). The main aim of the work is to identify spelling errors and their causes at B2 level, as well as to compare the total number of spelling errors made by two groups of learners at different levels - A2 and B2. The analysis has shown that the most frequent errors are those of the substitution of the consonants $\sigma-\beta[\mathrm{b}]-[\mathrm{v}]$, the vowels $u$-bl [i]-[i], and the soft sign. The cause of the errors is explained by the differences between Spanish and Russian phonological
\end{abstract}

\begin{abstract}
Me gustaría agradecer la colaboración de la Escuela Oficial de Idiomas de A Coruña, especialmente a Irina Stepánova Bolshakova, por la ayuda en la recogida del corpus, así como a todos los estudiantes que hayan querido participar en el estudio. Quiero dar las gracias a los dos revisores anónimos por los comentarios críticos de la versión anterior de este artículo. Asimismo, agradezco la financiación de la Consellería de Cultura, Educación e Ordenación Universitaria da Xunta de Galicia, ED481A-2017/279).
\end{abstract}

Para citar este artículo: Ogneva, Anastasiia (2021). ¿Cómo escriben los aprendices hispanohablantes de nivel intermedio en ruso como LE? Un estudio sobre la influencia fonológica de L1 en LE. ELUA, 35: 203-220. https://doi.org/10.14198/ELUA2021.35.10

Recibido: 17/04/2020, Aceptado: 23/10/2020

(C) 2021 Anastasiia Ogneva

Este trabajo está sujeto a una licencia de Reconocimiento 4.0 Internacional

de Creative Commons (CC BY 4.0) 
tes entre los sistemas fonológicos de español y ruso. A pesar de que no se han encontrado diferencias significativas entre los corpus de los aprendices de niveles B2 y A2, se observa que los errores ortográficos disminuyen según vaya avanzando el nivel de los aprendices.

PALABRAS CLAVE: análisis de errores; aprendizaje de lenguas extranjeras; ruso; ortografía; fonología. systems. Although no significant differences have been found between the corpus of B2 and A2 learners, it is observed that spelling errors decrease, as the learners advance.

KEYWORDS: error analysis; second language acquisition; Russian; orthography; phonology.

\section{INTRODUCCIÓN}

Una de las competencias clave del dominio de un idioma extranjero es la habilidad para expresarse e interactuar de forma escrita, que es algo indispensable para llevar a cabo la comunicación requerida en ciertos ámbitos. Por ejemplo, en el ámbito académico es tan importante comunicarse bien de forma oral como lo es escribir sin errores que puedan inhibir la comprensión del mensaje. Los errores que se cometen en el proceso de aprendizaje y que forman parte de la interlengua de los aprendices (Selinker 1972, 2013, 2014) constituyen una prueba de que el proceso de aprendizaje está avanzando. Por lo tanto, resulta importante explorar el tipo de errores que suelen cometer los aprendices de una lengua extranjera (LE) para poder desarrollar métodos didácticos eficientes que puedan prevenir estos errores y ayudar a utilizar el lenguaje escrito de manera apropiada.

Uno de los modelos que se emplean en la adquisición de idiomas extranjeros es el análisis contrastivo (AC) que propone comparar la lengua materna del alumnado con la lengua meta con el objetivo de revelar sus diferencias y semejanzas. De esta manera, es posible predecir los problemas que surgen a lo largo del aprendizaje, determinar las técnicas de instrucción o preparar el material didáctico (Fries 1945; Lado 1957). El análisis de errores (AE) es otra metodología a la que recurren muchos estudios en el campo de la adquisición de segundas lenguas. Esta técnica surgió a finales de los años 60 (Corder 1967, 1975, 1981; Muñoz-Basols y Bailini 2018) y proponía estudiar los errores que cometen los aprendices de lenguas extranjeras con el fin de descubrir qué estrategias utilizan los alumnos en el proceso de aprendizaje y las causas de sus errores (Fernández 1997). Frecuentemente los errores están asociados con la "transferencia negativa" o "interferencia" que es el proceso de utilizar las diferentes estructuras gramaticales y el vocabulario de la primera lengua (L1) en la LE (Fries 1945; Lado 1957). Asimismo, cuando la L1 y la LE de los aprendices comparten algunas características gramaticales, se produce la "transferencia positiva", dado que los aprendices utilizan los aspectos de su L1 que también son correctos en la LE.

El objetivo del presente estudio es identificar los errores ortográficos que cometen los aprendices hispanohablantes de ruso como LE de nivel intermedio (B2) en relación con los sistemas fonológicos de la L1 y la LE y, de este modo, ampliar los hallazgos de las investigaciones anteriores. En concreto, se plantea alcanzar dos objetivos. En primer lugar, analizar un corpus de 30 trabajos escritos por aprendices de nivel B2 en términos de errores ortográficos y, en segundo lugar, realizar comparaciones con otro corpus de trabajos escritos 
por aprendices de nivel A2 (Ogneva 2018). El presente trabajo se estructura de la siguiente manera. El apartado 2 trata de abordar algunos estudios previos llevados a cabo con aprendices hispanohablantes de ruso. La tercera parte se centra en una revisión de los sistemas ortográficos y fonológicos en español y en ruso. En el apartado 4 se explica el presente estudio, describiendo el corpus de trabajos escritos recopilado y la metodología de análisis empleada. El apartado 5 describe los resultados cuantitativos de los aprendices del nivel B2 y los compara con los aprendices del nivel A2. En el apartado 6 se describe el análisis cualitativo de los errores ortográficos en el nivel B2. Finalmente, en el apartado 7 se exponen las conclusiones y se proponen algunas líneas para futuras investigaciones.

\section{EL APRENDIZAJE DEL RUSO POR APRENDICES HISPANOHABLANTES: LOS ESTUDIOS PREVIOS}

Los estudios que han investigado el aprendizaje del ruso por aprendices hispanohablantes y, especialmente, los errores ortográficos, no son muy numerosos e incluyen investigaciones centradas en el análisis contrastivo entre ruso y español y en el análisis de errores cometidos por los aprendices hispanohablantes de ruso LE.

En la línea del análisis contrastivo, el primer estudio que se llevó a cabo fue el de Vinogradov y Miloslavskiy (1979a, 1979b, 1980, 1986) quienes compararon los sistemas morfológicos del español y del ruso. Así, por ejemplo, indicaron que el sistema morfológico del número es relativamente semejante, siendo el sistema ruso más complejo, dada la variedad de terminaciones que se añaden a los sustantivos al formar el plural. Otras investigaciones de carácter contrastivo incluyen los trabajos de Guzmán Tirado y Herrador Pina (2000), Guzmán Tirado y Quero Gervilla (2003) y Guzmán Tirado (2007). Asimismo, Quero Gervilla (2004, 2005), Denissenko (2016) y Popova (2007) estudiaron otros aspectos del aprendizaje del ruso por parte de hispanohablantes. Por ejemplo, la adquisición del sistema de declinaciones (casos), el desarrollo del proceso de aprendizaje global de ruso en tres niveles, así como los verbos modales "poder" y "querer" en español y en ruso.

En lo que se refiere a la correlación entre el componente fonológico y ortográfico, los estudios previos llevados a cabo con aprendices de inglés como L2 demuestran que los errores ortográficos ocurren cuando existe una incongruencia entre fonemas en la L1 y L2 (Cook 1997; Fashola et al. 1996; Park 2011). Por ejemplo, Ibrahim (1978) examinó los trabajos escritos de los aprendices árabes de inglés y concluyó que las diferencias existentes entre los sistemas fonológicos afectaban la ortografía de los aprendices en inglés L2. Así, se observó la sustitución del fonema [p] por el [b], puesto que en árabe no existe el fonema [p] (p.ej., bicrure en lugar de picture 'dibujo', hapit en vez de habit 'hábito'). Los hallazgos similares se encontraron en el estudio de Sun-Alperin y Wang (2008) con niños y niñas hispanohablantes que aprendían inglés como L2. En concreto, los errores observados se explican por la influencia del componente fonológico del español en la escritura de inglés L2 (p.ej., *mun en vez de moon 'luna'). Del mismo modo, las autoras sugieren que el nivel de la transparencia ortográfica influye en el desarrollo de la escritura en inglés L2. Es decir, los participantes hispanohablantes tenían más dificultad en la escritura de vocales en inglés cuando estas no tenían una correspondencia directa entre fonema y grafema. Siguiendo la misma línea de investigación, Park (2011) comparó la escritura de pseudopalabras en inglés entre los hablantes nativos de este idioma y los nativos de coreano que aprendían el inglés 
como L2. Las pseudopalabras que componían la prueba eran de dos tipos: por un lado, la condición congruente incluía aquellos ítems que contenían fonemas existentes en ambas lenguas (p.ej., [m] o [n]) y, por otro lado, la condición incongruente estaba compuesta por los ítems que contenían fonemas no existentes en coreano (p.ej., [f] o [v]). Sus resultados reflejan que los participantes coreanos presentaban más dificultad con las pseudopalabras de la condición incongruente, es decir, que incluían fonemas que no existen en coreano.

Llevando a cabo un análisis contrastivo, Bondarenko y Loginova (2014) estudiaron el sistema fonológico ruso y lo compararon con los sistemas fonológicos italiano y español. En sus conclusiones se recogen los aspectos más complejos de la fonología rusa para los aprendices nativos de lenguas romances, entre ellos, la acentuación de las palabras y los diferentes sonidos vocálicos no presentes en dichas lenguas (p.ej., $b l[\mathrm{i}], ю[\mathrm{H}], я[æ]$ ). Además, las investigadoras indican que el proceso de reducción vocálica no es percibido por los nativos de lenguas romances, dado que sus lenguas nativas carecen de este proceso fonológico. Klimova et al. (2017) compararon los sistemas fonológicos del ruso y del español e identificaron las principales diferencias entre ambos. Entre ellas, se encuentran la distinción entre sonidos duros y blandos en ruso, así como una serie de sonidos rusos inexistentes en español. También se mencionan las diferencias referentes a la acentuación de las palabras en ambas lenguas.

Golikov (2008) fue el primero en investigar las dificultades de los aprendices hispanohablantes en el nivel ortográfico a la hora de aprender ruso. En concreto, en su trabajo el investigador concluyó que las letras que se escriben de manera semejante, por ejemplo, $u$, $u, ~ \iota, u$ (pero tienen una representación fonológica distinta) resultan ser muy complicadas para los hispanohablantes, que a menudo se confunden a la hora de representarlas en la escritura. Por su parte, Ohalina $(2018)^{1}$ mediante el análisis de errores investigó las dificultades que tienen los aprendices hispanohablantes procedentes de Ecuador y Colombia en la fonología rusa. Sus resultados reflejan que los problemas principales son la incapacidad para diferenciar entre los sonidos [b] y [v] y entre los sonidos sordos y sonoros [s]-[z] y [s]-[z]. Del mismo modo, se apunta que el acento resulta complicado para los aprendices hispanohablantes, dado que no existen reglas específicas sobre la acentuación de las palabras y, sin embargo, el cambio en el acento de una palabra puede modificar el significado (p.ej., замо́к /zemok/ 'cerradura' у за́мок/zamək/ 'castillo')2.

Los estudios previos enfocados en el análisis profundo del corpus de trabajos escritos por aprendices de nivel A2 (Ogneva 2018, 2019) y, en concreto, en el análisis de errores ortográficos, indica que los alumnos del nivel básico presentan dificultad con el uso de algunas consonantes (entre ellas, $\sigma-\beta[\mathrm{b}]-[\mathrm{v}]$ y $c-3[\mathrm{~s}]-[\mathrm{z}]$ ) y algunas vocales (p.ej., $u-b l$ [i]-[i] , $e-u$ [e]-[i], $a-я$ [a]-[æ]). De manera semejante, se identificaron numerosos errores en el uso del signo blando (p.ej., *факултет /fakultet/ en vez de факультет /fakul'tet/, *неболшой /nebolšoj/ en lugar de небольшой/nebol'šoj/. Es decir, parece que los aprendices del nivel A2 no distinguen los sonidos palatalizados y no palatalizados y, por lo tanto, lo expresan incorrectamente en la escritura.

Resumiendo, las investigaciones previas indican que, dadas las diferencias entre los sistemas fonológicos y ortográficos ruso y español, los aprendices hispanohablantes de ruso

\footnotetext{
1 Los resultados de este trabajo se tienen que considerar con cautela, puesto que en su versión publicada no se específica ni el número de los aprendices que han participado ni la cifra exacta de los errores cometidos en cada categoría.

2 La transliteración de caracteres cirílicos se efectúa mediante la norma internacional ISO 9 (1995).
} 
como LE presentan ciertas dificultades con el manejo del sistema ortográfico de la lengua meta. Estas dificultades se explican mayoritariamente por la influencia del sistema fonológico de la L1 de los alumnos. De esta manera, resulta imprescindible que los aprendices de LE desarrollen la conciencia fonológica que se refiere a "la toma de conciencia de cualquier unidad fonológica del lenguaje hablado" (Jiménez González y Ortiz González 1995: 23) y también la conciencia ortográfica que consiste en ser consciente de la correspondencia existente entre grafemas y fonemas (Kirk y Gillon 2009).

\section{LOS SISTEMAS ORTOGRÁFICOS EN RUSO Y EN ESPAÑOL}

Para poder identificar los errores ortográficos que cometen los aprendices hispanohablantes de ruso como LE es necesario señalar las principales diferencias existentes entre los dos sistemas ortográficos y fonológicos.

El ruso y el español son lenguas de dos familias lingüísticas distintas. Por lo tanto, los sistemas ortográficos son diferentes. En ruso se utiliza el alfabeto cirílico de origen griego que consta de 33 letras ( 21 consonantes, 10 vocales y 2 signos), mientras que en español se emplea el alfabeto latino compuesto por 27 letras. Algunas letras se escriben igual en español y en ruso, por ejemplo, A-a, O-o, E-e. Otras letras se escriben de la misma manera solo en mayúsculas $(\mathrm{M}, \mathrm{K}, \mathrm{T})$, presentando diferencias en la escritura en minúsculas (compare м - m, к - k у т - t). En cuanto a las diferencias entre ambos sistemas ortográficos, podemos destacar el hecho de que a pesar de que algunas letras se escriben de manera similar en español y en ruso, estas representan sonidos diferentes. Entre ellas se encuentran la $y$ ([ซ] en ruso, [i] en español) y la $u$ ([i] en ruso, [u ] en español). Por lo tanto, los aprendices confunden la letra $y$ que corresponde al sonido [ $ซ$ ] en ruso con la letra $i$ griega que se escribe de manera similar y corresponde al sonido [i] en español y viceversa. Así, se observan casos como *дриг /drig/ en lugar de друг /drug/.

Una de las complejidades del sistema ortográfico ruso consiste en que el alfabeto cirílico manuscrito presenta ciertas diferencias en comparación con el alfabeto tipográfico. En la Figura 1 se pueden observar ambos alfabetos y sus respectivas diferencias. Así, por ejemplo, la letra Д- $\partial(/ \mathrm{de} /)$ se escribe de forma distinta en ambos alfabetos.

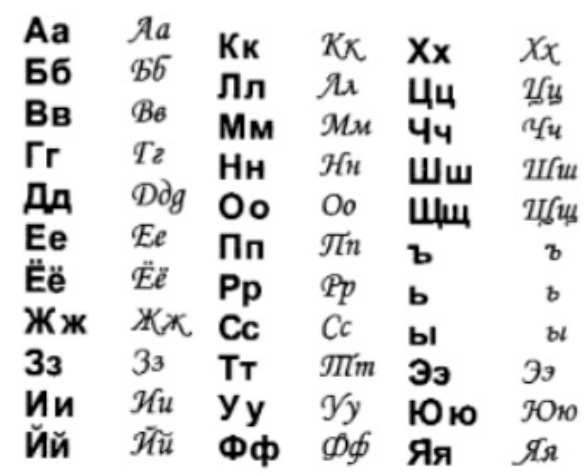

Figura 1. El alfabeto cirílico ruso. En negrita aparecen las letras tipográficas y en cursiva las letras manuscritas. Adaptado de Sánchez Puig et al. (2005) 
El español y el ruso se diferencian en lo que se refiere al grado de transparencia ortográfica. En español la correspondencia entre el fonema y el grafema que componen una palabra es relativamente directa y consistente. Así, la letra A se pronuncia siempre como [a], independientemente de la palabra: mes $a$, casa, más (Míguez Álvarez 2018). No obstante, en las lenguas opacas (como, por ejemplo, el ruso), la relación entre los grafemas y los fonemas no es tan clara y, en algunas ocasiones, presenta irregularidades. Por ejemplo, a un mismo grafema le corresponden varios sonidos o que un sonido posea varios grafemas (Denes 2011). De esta manera, la letra A se puede pronunciar de distintas formas dependiendo de su posición en la palabra, p.ej., sobáka /sebakə/. En este ejemplo, además, observamos un caso de reducción vocálica en el que la letra $\mathrm{O}$, dada su posición átona en la palabra, se convierte en [e] (Guzmán Tirado y Herrador del Pino 2002; Dmítrieva 2017). En la Tabla 1 se recoge la información contrastiva entre los dos sistemas ortográficos y fonológicos.

Respecto al signo blando y signo duro, que a menudo provocan confusión entre los aprendices de ruso como LE, estos cumplen varias funciones. Una de ellas es la función de separación entre las vocales $(e[\mathrm{e}], \ddot{e}[\Theta], ю[\mathrm{H}], я[æ])$ y las consonantes después de prefijos. En esencia, al utilizar el signo blando o duro lo que sucede es que se separa la vocal de la consonante, provocando una breve pausa en la pronunciación (Sánchez Puig et al., 2005). Compare, por ejemplo, сел /sel/ 'él se sentó' у съел /s"el/ 'él comió'. Por otro lado, el signo blando sirve para palatalizar las consonantes a través de un proceso de palatalización. En ruso empleando el signo blando se puede palatalizar cualquier consonante (excepto la $\varphi$ $\left[\widetilde{\mathrm{t}} \mathrm{e}^{\prime}\right], u[\mathrm{~s}]$ y $m$ [6:] que siempre son blandas). Así, se puede observar un cambio importante en el significado entre dos palabras, cuya única diferencia es la presencia del signo blando. Por ejemplo, угол /ugol/ 'rincón' о 'ángulo' у уголь /ugol'/ 'carbón' о браm /brat/ 'hermano' y брamb /brat'/ 'coger' (Sánchez Puig et al. 2005).

\begin{tabular}{|c|c|c|c|}
\hline Letra en ruso & Representación fonética & Letra en español & Representación fonética \\
\hline \multicolumn{4}{|c|}{ Vocales } \\
\hline A-a & [a], [e], [ə] & A-a & {$[\ddot{a}]$} \\
\hline E-e & {$[\mathrm{e}]$} & - & - \\
\hline Ë-ë & {$[\Theta]$} & - & - \\
\hline И-и & [i] & $\mathrm{I}-\mathrm{i}$ & [i] \\
\hline O-o & [o] & O-O & [o] \\
\hline $\mathrm{y}-\mathrm{y}$ & {$[\sigma]$} & U-u & {$[\mathrm{u}]$} \\
\hline Ы-ы & [i] & - & - \\
\hline Э-э & {$[\varepsilon]$} & E-e & [et] \\
\hline Ю-ю & {$[\mathrm{H}]$} & - & - \\
\hline Я-я & {$[æ]$} & - & - \\
\hline \multicolumn{4}{|c|}{ Consonantes } \\
\hline Б-б & {$[\mathrm{b}],\left[\mathrm{b}^{\mathrm{j}}\right]$} & B-b & {$[\mathrm{b}],[\beta]$} \\
\hline B-в & {$[\mathrm{v}],\left[\mathrm{v}^{\mathrm{j}}\right]$} & $\mathrm{V}-\mathrm{v}$ & {$[\mathrm{b}],[\beta]$} \\
\hline$\Gamma-\Gamma$ & {$[\mathrm{g}],\left[\mathrm{g}^{\mathrm{j}}\right],[\gamma]$} & G-g & {$[\mathrm{g}],[\mathrm{\gamma}]$} \\
\hline Д-д & {$[\mathrm{d}],\left[\mathrm{d}^{\mathrm{j}}\right]$} & D-d & [d], [ơ] \\
\hline Ж-ж & {$[\mathrm{z}]$} & - & - \\
\hline $3-3$ & {$[\mathrm{z}],\left[\mathrm{z}^{\mathrm{j}}\right]$} & - & - \\
\hline
\end{tabular}




\begin{tabular}{|c|c|c|c|}
\hline Й-й & [j] & - & - \\
\hline К-к & {$[\mathrm{k}],\left[\mathrm{k}^{\mathrm{j}}\right]$} & K-k & {$[\mathrm{k}]$} \\
\hline Л-л & {$[1],\left[\mathrm{l}^{\mathrm{j}}\right]$} & L-1 & {$[1],[1],[1 \mathrm{l}],[1],[1]$} \\
\hline M-M & {$[\mathrm{m}],\left[\mathrm{m}^{\mathrm{j}}\right]$} & M-m & {$[\mathrm{m}],[\mathrm{m}]$} \\
\hline Н-н & {$[\mathrm{n}],\left[\mathrm{n}^{\mathrm{j}}\right]$} & $\mathrm{N}-\mathrm{n}$ & {$[\mathrm{n}],[\mathrm{n}],[\mathrm{n}],\left[\mathrm{n}^{\mathrm{j}}\right],[\mathrm{n}]$} \\
\hline П-П & {$[\mathrm{p}],\left[\mathrm{p}^{\mathrm{j}}\right]$} & P-p & {$[\mathrm{p}]$} \\
\hline P-p & {$[\mathrm{r}],\left[\mathrm{r}^{\mathrm{j}}\right]$} & R-r & {$[\mathrm{r}],[\mathrm{r}]$} \\
\hline $\mathrm{C}-\mathrm{c}$ & {$[\mathrm{s}],\left[\mathrm{s}^{\mathrm{j}}\right]$} & S-s & {$[\mathrm{s}],[\mathrm{z}]$} \\
\hline $\mathrm{T}-\mathrm{T}$ & {$[\mathrm{t}],[\mathrm{t}]$} & $\mathrm{T}-\mathrm{t}$ & {$[\mathrm{t}]$} \\
\hline$\Phi-\phi$ & {$[\mathrm{f}],[\mathrm{f}]$} & F-f & [f] \\
\hline$X-x$ & {$[\mathrm{x}],\left[\mathrm{x}^{\mathrm{j}}\right]$} & $\mathrm{J}-\mathrm{j}$ & {$[\mathrm{x}],[\chi]$} \\
\hline Ц-ц & [ts] & - & - \\
\hline Ч-ч & {$\left[\widehat{t} 6^{\prime}\right]$} & - & - \\
\hline Ш-ш & {$[\mathrm{s}]$} & - & - \\
\hline Щ-щ & [6:] & - & - \\
\hline \multicolumn{4}{|c|}{ Signos } \\
\hline ь (signo blando) & , & - & - \\
\hline ь (signo duro) & " & - & - \\
\hline
\end{tabular}

Tabla 1. Diferencias y semejanzas entre los alfabetos empleados en ruso y español. Las representaciones fonológicas se adaptan del Alfabeto Fonético Internacional (IPA 1999)

En resumen, existen ciertas diferencias y similitudes entre los sistemas fonológicos y ortográficos de ambas lenguas. Conforme con las teorías de transferencia positiva de los aspectos de la L1 a los de la LE, suponemos que las semejanzas entre los sistemas fonológicos y la escritura de algunas letras ayudarán a los aprendices a utilizar con más facilidad el sistema ortográfico de la LE. Por otro lado, las diferencias fonológicas y ortográficas entre ambas lenguas provocarán errores de forma frecuente.

\section{EL PRESENTE ESTUDIO}

\subsection{Contexto del estudio}

Los trabajos escritos que forman parte del corpus que se analiza en el presente estudio se recogieron en la Escuela Oficial de Idiomas (EOI) de A Coruña. La EOI es una institución de formación no reglada que se centra en la enseñanza de idiomas extranjeros. La enseñanza en la EOI está estructurada en cuatro niveles: básico 1 (equivale a nivel A1 del Marco Común Europeo de Referencia para las Lenguas (MCER)), básico 2 (nivel A2 del MCER), intermedio (nivel B1 del MCER) y avanzado (nivel B2 del MCER). Las clases tienen una duración de dos horas y se celebran dos veces a la semana. Los alumnos que obtienen el nivel B2 han recibido entre 500 y 600 horas de formación presencial, según la programación propia de la EOI. Según el MCER (2002: 26), el aprendiz que alcance dicho nivel:

Es capaz de entender las ideas principales de textos complejos que traten de temas tanto concretos como abstractos, incluso si son de carácter técnico, siempre que estén dentro 
de su campo de especialización. Puede relacionarse con hablantes nativos con un grado suficiente de fluidez y naturalidad, de modo que la comunicación se realice sin esfuerzo por parte de los interlocutores. Puede producir textos claros y detallados sobre temas diversos, así como defender un punto de vista sobre temas generales, indicando los pros y los contras de las distintas opciones.

En cuanto a la escritura, en el MCER (véase Tabla 2) se especifican diferentes tipos de destreza de expresión escrita, entre ellas, el dominio de la escritura creativa y de la escritura de informes y redacciones. Por otro lado, se menciona el dominio de la ortografía de la lengua meta.

Expresión escrita en general

Escribe textos claros y detallados sobre una variedad de temas relacionados con su especialidad, sintetizando y evaluando información y argumentos procedentes de varias fuentes (p. 64).

\section{Escritura creativa}

- Escribe descripciones claras y detalladas de hechos y experiencias reales o imaginarias en textos claros y estructurados, marcando la relación existente entre las ideas y siguiendo las normas establecidas del género literario elegido.

- Escribe descripciones claras y detalladas sobre una variedad de temas relacionados con su especialidad. Sabe escribir una reseña de una película, de un libro o de una obra de teatro (p.65).

Informes y redacciones

- Escribe redacciones e informes que desarrollan sistemáticamente un argumento, destacando los aspectos significativos y ofreciendo detalles relevantes que sirvan de apoyo. Sabe evaluar las diferentes ideas o soluciones que se pueden aplicar a un problema.

- Escribe redacciones o informes que desarrollan un argumento, razonando a favor o en contra de un punto de vista concreto y explicando las ventajas y las desventajas de varias opciones. Sabe sintetizar información y argumentos procedentes de varias fuentes (p. 66).

\section{Dominio de la ortografía}

Produce una escritura continua inteligible que sigue las convenciones de organización y de distribución en párrafos. La ortografía y la puntuación son razonablemente correctas, pero puede manifestar la influencia de la lengua materna (p.115).

Tabla 2. Las competencias de escritura que deben tener los aprendices del nivel B2, según MCER

En relación con la competencia ortográfica adquirida al alcanzar el nivel intermedio de ruso, se consultó la programación de la EOI de A Coruña. Conforme con esta, los alumnos deben ser capaces de producir patrones gráficos de uso común, generales y en entornos específicos (abreviaturas comunes, cursiva, asteriscos, paréntesis) con razonable corrección. En cuanto a los contenidos específicos, cabe destacar el dominio de los siguientes aspectos:

El alfabeto cirílico (manuscrito y tipográfico).

- Ortografía de las palabras extranjeras según su procedencia.

- El uso de las mayúsculas.

- El uso de signos ortográficos: guion, signos de puntuación, diéresis.

- El manejo de $A / O$ [a]-[o] y Е/И [e]-[i] en las raíces de los sustantivos sin acentuar.

- El manejo de He/Hи /ne-ni/, -HH- /nn/, -CC- /ss/ y -ЖЖ-/žž/. 
- Normas de formación de palabras.

- Uso de los caracteres en sus diferentes formas (siglas, puntos cardinales, siglos, nombres abstractos, organismos públicos).

- Ortografía de adverbios y uso de guion.

- Normas de transliteración del ruso al castellano y viceversa.

\subsection{Descripción del corpus}

El corpus se compone de un total de 80 trabajos escritos. En concreto, son 30 redacciones de aprendices de nivel B2 que se analizan en el presente estudio y 50 trabajos de aprendices de nivel A2 que se analizaron en una investigación previa. En ambos casos se trata de muestras de expresión escrita producto de las actividades realizadas a lo largo del curso académico (2015-2016 en el nivel A2, 2018-2019 en el nivel B2) y del examen final. Las producciones escritas son de tipo redacciones e informes, según el MCER, y los tópicos descritos incluían las fiestas de Navidad en Rusia y en España, los objetos que actualmente no se utilizan, cartas a un amigo/a que quiere hacer un curso de español, etc.

La longitud media de los textos de los aprendices de nivel A2 es de 90 palabras y la de los textos de los aprendices de nivel B2 es de 258. Los grupos de estudiantes cuyos trabajos forman parte del corpus es heterogéneo: se incluyen las redacciones tanto de mujeres como de hombres de edades comprendidas entre los 30 y 65 años. Los alumnos poseían experiencias diferentes en términos de aprendizaje de lenguas extranjeras, así como distintos niveles educativos y profesiones.

\subsection{Metodología}

El objetivo de este trabajo es identificar los errores ortográficos cometidos por los aprendices hispanohablantes de ruso como L2. Por lo tanto, en la fase preliminar clasificamos los errores según el tipo: errores de sustitución, errores de omisión, errores de inserción, uso de letras latinas y errores poco habituales. El sistema de clasificación fue parcialmente adaptado de Cook (1997).

Durante la primera fase de compilación del corpus se realizó un conteo y una clasificación manual de los errores. El análisis cuantitativo se llevó a cabo con el programa Statistical Package for Social Sciences versión 25 y se describe a continuación en el apartado 5. El apartado 6 se dedica al análisis cuantitativo de cada tipo de error cometido por los aprendices hispanohablantes de nivel B2 de ruso como L2.

\section{RESULTADOS CUANTITATIVOS}

En primer lugar, se ha llevado a cabo un análisis cuantitativo del número de errores en su totalidad y según su categoría. En total, el análisis ha reflejado 187 errores ortográficos, de los cuales 125 son errores de sustitución, 30 son errores de omisión, 13 son errores de inserción y 19 se corresponden con otro tipo de errores (uso de letras latinas y errores poco habituales). La media de errores por oración es de 6,2. El resumen de los errores por categoría se encuentra en la Figura 2. 


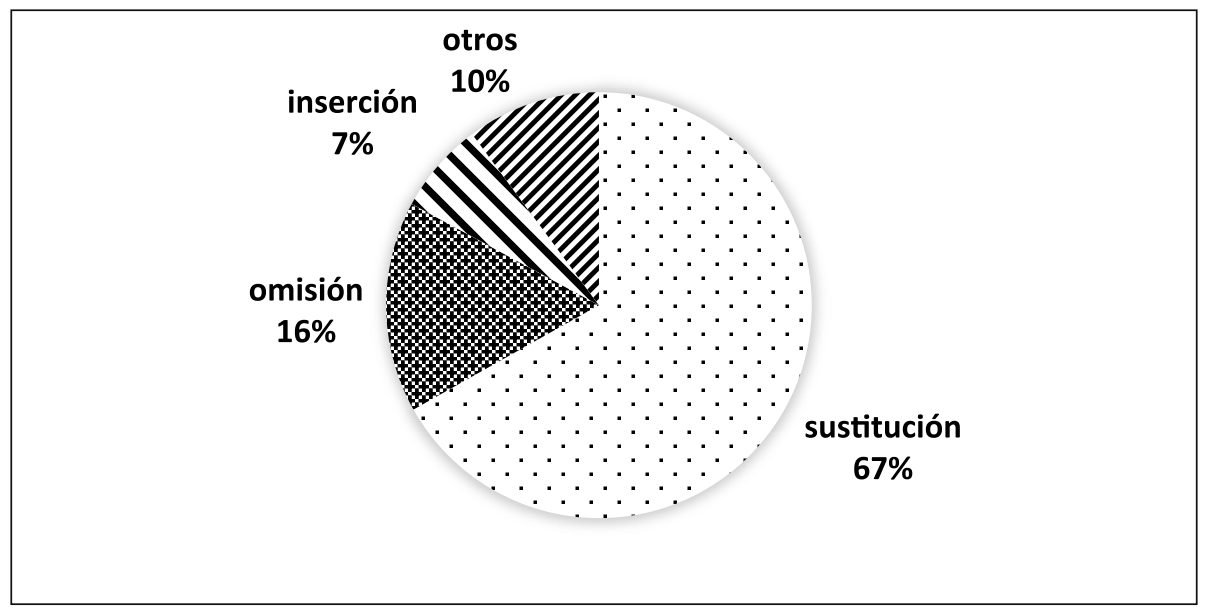

Figura 2. Errores por categoría cometidos por los aprendices del grupo B2 (expresados en porcentajes)

A continuación, dentro de la categoría de errores de sustitución, se distinguen tres tipos de errores: errores de sustitución de consonantes, de vocales y errores con el signo blando. Casi la mitad de los errores de sustitución corresponde al grupo de vocales (48\%), frente a un $32,8 \%$ de errores correspondiente al grupo de consonantes y un 19,2\% correspondiente al grupo de errores con el signo blando. En la Figura 3 se encuentra el número total de los errores de sustitución y el número de los errores más frecuentes en esta categoría.

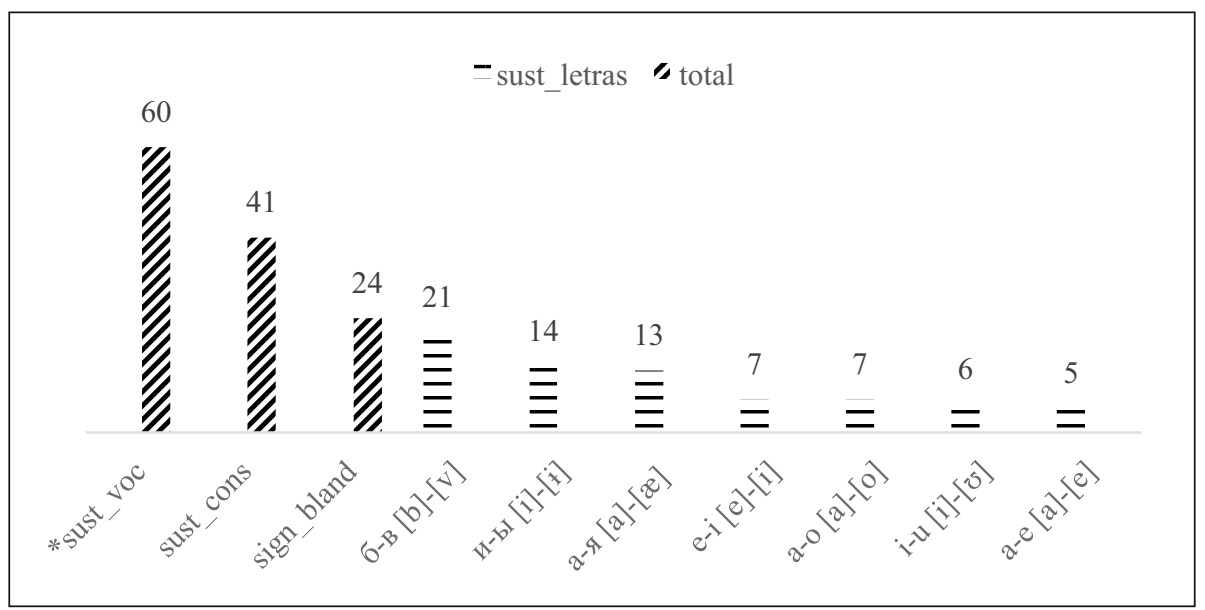

Figura 3. Errores de sustitución por categoría (total) y por frecuencia. *sust_voc: sustitución de vocales, sust_cons: sustitución de consonantes, sign_bland: sustitución de signo blando

A continuación, se han realizado las comparaciones entre los grupos A2 y B2. El número total de errores en el nivel A2 es de 294, mientras que en el nivel B2 se disminuye hasta 
187 errores. El análisis estadístico reflejó que las diferencias entre el número total de errores en diferentes niveles no son significativas $(p>0,05)$. En el caso de sustitución de $6-\beta[\mathrm{b}]-$ [v], la tendencia indica que los aprendices de nivel más avanzado cometen menos errores. Se observa, además, una disminución de errores en total (294 en el nivel A2 y 187 en el nivel B2) y por grupos de errores. Así, los errores se sustitución han bajado de 213 a 125, los de omisión han descendido de 37 a 30 y los de inserción han disminuido de 32 a 13 . El único grupo de errores que ha subido es el grupo de otros errores que incluye los casos poco habituales y el uso de letras latinas (de 12 en el nivel A2 a 19 en el nivel B2). Estos resultados se resumen en la Figura 4.

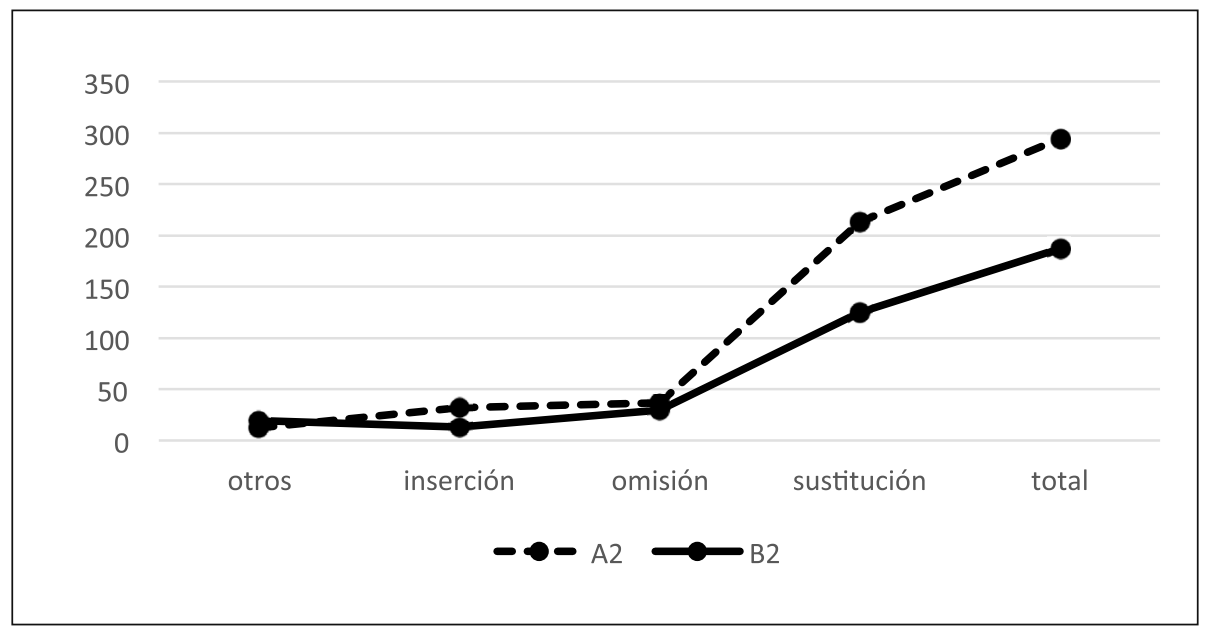

Figura 4. Errores cometidos por los aprendices en el nivel A2 y en el nivel B2. La línea continua indica el nivel B2; la línea descontinua corresponde al nivel A2

En la Tabla 3 se puede observar la comparación entre los grupos A2 y B2 en relación con los errores de sustitución cometidos en total y los más frecuentes. El análisis demuestra que los aprendices del nivel A2 cometen más errores con la sustitución de consonantes. En concreto, cometieron 106 errores, que constituye casi la mitad de los errores de sustitución, mientras que los aprendices del nivel B2 cometieron 41 errores (32,8\% del total). Respecto a la sustitución de consonantes, el error más frecuente en ambos niveles es la sustitución de la letra $\sigma[\mathrm{b}]$ por la $\theta[\mathrm{v}]$ o viceversa. Otro error que se repite en los dos niveles con relativa frecuencia es la sustitución de la $c$ [s] por la 3 [z] y viceversa. Se identificaron 7 errores de este tipo en el nivel A2 y 4 en el nivel B2. En relación con los errores de sustitución de vocales, el análisis refleja que los aprendices del nivel B2 cometen más errores de este tipo (60 errores, un 48\% del total) que los aprendices del nivel A2 (75 errores, un 35,21\% del total). En cuanto a los errores relacionados con el uso del signo blando, el análisis refleja que en el nivel B2 los aprendices cometen un mayor número de errores $(19,2 \%$ en comparación con $15,02 \%$ en el nivel A2). 


\begin{tabular}{lcc}
\hline & $\mathrm{A} 2$ & $\mathrm{~B} 2$ \\
\hline Sustitución de consonantes & $106(49,77 \%)$ & $41(32,8 \%)$ \\
\hline$\sigma-\boldsymbol{b}[\mathrm{b}]-[\mathrm{v}]$ & $44(20,66 \%)$ & $21(16,8 \%)$ \\
\hline $3-c[\mathrm{z}]-[\mathrm{s}]$ & $7(3,29 \%)$ & $4(3,2 \%)$ \\
\hline Sustitución de vocales & $75(35,21 \%)$ & $60(48 \%)$ \\
\hline$u-b l[\mathrm{i}]-[\mathrm{i}]$ & $25(11,74 \%)$ & $14(11,2 \%)$ \\
\hline$a-o[\mathrm{a}]-[\mathrm{o}]$ & $19(8,92 \%)$ & $7(5,6 \%)$ \\
\hline$e-u[\mathrm{e}]-[\mathrm{i}]$ & $16(7,51 \%)$ & $7(5,6 \%)$ \\
\hline$a-я[\mathrm{a}]-[æ]$ & $13(6,10 \%)$ & $13(10,4 \%)$ \\
\hline Signo blando & $32(15,02 \%)$ & $24(19,2 \%)$ \\
\hline Errores de sustitución en total & $213(100 \%)$ & $125(100 \%)$ \\
\hline
\end{tabular}

Tabla 3. Comparación de los errores de sustitución (totales y los más frecuentes) entre A2 y B2

\section{RESULTADOS CUALITATIVOS}

Los resultados cualitativos se presentarán a continuación atendiendo a cada categoría de error. En primer lugar, analizaremos los errores de sustitución, que incluyen la sustitución de consonantes, la de vocales y los errores con el signo blando. Posteriormente, se expondrán los errores de omisión e inserción. Por último, se describirá el grupo de otros errores, que incluye el uso de letras latinas y los casos de errores poco habituales.

\subsection{Errores de sustitución}

Los errores de sustitución, como se ha explicado en el apartado anterior, son los más frecuentes y constituyen un $66,84 \%$ de los errores en total (125 de 187). A continuación, se ofrece un análisis descriptivo de cada subtipo de esta categoría.

\subsubsection{Consonantes}

La mayoría de los errores de sustitución están relacionados con el sistema fonológico tanto de la L1 de los alumnos como de la propia LE. En total, se identificaron 41 errores de sustitución de consonantes $(32,8 \%)$. El error más frecuente de sustitución de consonantes es el intercambio de las letras $\sigma[\mathrm{b}]$ у $в$ [v]. Este resultado indica que, dado que en español los grafemas b y v están representados por el mismo sonido [b] o [ $\beta]$ ] (según su posición en la palabra), los hablantes hispanohablantes no consiguen realizar la distinción entre estos fonemas en ruso $\left([\mathrm{b}] \mathrm{y}[\mathrm{v}]\right.$ o $\left[\mathrm{b}^{\mathrm{j}}\right] \mathrm{y}\left[\mathrm{v}^{\mathrm{j}}\right]$ ). Por lo tanto, la representación grafémica en ruso LE se encuentra afectada. Asimismo, se observan dificultades en el uso de letras $c$ [s] y 3 [z]. A pesar de que la fricativa [s] se convierte en una sonora [z] en la posición antes de una consonante sonora como, por ejemplo, en rasgo /razyo/ (Hualde 2005; Martínez-Celdrán, Fernández-Planas y Carrera-Sabaté 2003), los aprendices hispanohablantes presentan una gran dificultad para la diferenciación de estos dos sonidos en ruso LE y, por tanto, confunden sus representaciones grafémicas. Los ejemplos 1-5 ilustran este tipo de error. 
(1) * вblвaеm /vyvaet/ (t2) en lugar de бывает /byvaet/ 'pasa'.

(2) *удовно /udovno/ (t6) en lugar de удобно /udobno/ 'cómodo'.

(3) *во-пербых /vo-perbyx/ (t8) en vez de во-первых /vo-pervyx/ 'en primer lugar'.

(4) *собирать обощей /oboŝej/ (t14) en vez de собирать овощи /sobirat' ovoŝi/ 'recoger verduras'.

(5) *изключением /izključeniem/ (t23) en lugar de исключением/isključeniem/ 'excepción'.

Otros errores de sustitución de consonantes son, por ejemplo, el intercambio de las letras que tienen una escritura semejante, $u$ [s] у $u$ [6:] (ejemplo 6) у $м$ [m] у $m$ [t] (ejemplo 8); y la confusión entre los sonidos sonoros y sordos, $\varkappa_{[\mathrm{z}]}$ y $u$ [s] (ejemplo 7) que no tienen una representación fonológica directa en español.

(6) *yame /čaše/ (t1) en lugar de yame /čaŝe/ 'más frecuente'.

(7) *dame/daše/ (t21) en lugar de даже /daže/) 'incluso'.

(8) *сетьёй /set'ëj/ (t23) en lugar de семьёй /sem'ëj/ 'como familia'.

\subsubsection{Vocales}

Los errores de sustitución de vocales constituyen un 48\% (60 errores) de todos los errores de sustitución, siendo por tanto los más numerosos. El error más frecuente (14 casos) es el intercambio de las letras $u$ [i] y bl [i], que constituye un 11,2\% del total. La articulación de ambos sonidos que representan estas letras es semejante en ruso. No obstante, la letra $b l$ y su correspondiente sonido [i] no existe en español, lo cual provoca confusión entre los aprendices de ruso LE y, por lo tanto, estos tienden a sobregeneralizar este sonido y su representación grafémica a la letra $u$ [i]. Algunos ejemplos se presentan a continuación.

(9) на *илощчады /na ploŝady/ (t1, t20) en lugar de на площ̧ади /na ploŝadi/ 'en la plaza'.

(10) *давные /davnye/ (t3) en lugar de давние /davnije/ 'antiguos'.

(11) *лампи /lampi/ (t16) en lugar de лампы /lampy/ 'lámparas'.

Otro error frecuente, que constituye un 10,4\% del total (13 casos) es la confusión entre las letras $a$ [a] у $я$ [æ], cuyas representaciones fonológicas también comparten la misma articulación. Los aprendices del nivel B2, por tanto, siguen cometiendo fallos en este aspecto, como se puede observar en los ejemplos 12-14.

(12) в *кровотах /v krovotah/ (t14) en lugar de в кроватяx /v krovatâh/ 'en las camas'.

(13) украшают * гирландами /girlandami/ (t20) en lugar de украшают гирляндами /girlândami/ 'se decora con guirlandas'.

(14) *nользуются фонарикями /fonarikâmi/ (t21) en lugar de пользуются фонариками / fonarikami/ 'utilizan las linternas'.

Además de los errores relacionados con la confusión entre sonidos que se articulan de manera semejante, se identificaron errores producidos como consecuencia de algunos procesos internos de la lengua rusa y los niveles de opacidad de esta. En concreto, se trata del proceso de reducción vocálica que convierte el sonido átono [o] en [a] o en [ə] (dependiendo de la posición en la palabra) y el sonido átono [e] en [i] (Guzmán Tirado y Herrador del Pino 2002; Dmítrieva 2017). Así, los errores de ambos tipos constituyen un 5,6\% del total, lo 
cual equivale a 7 errores (véanse ejemplos 15-18). Dentro del grupo de errores de o-a se han identificado los errores de hipercorrección (Quero Gervilla 2004), producidos cuando el aprendiz empleaba la $o$ en lugar de la $a$ (véase el ejemplo 19).

(15) * када (/kada/) (t9) en lugar de когда (/kogda/) 'cuándo'.

(16) * поговарить (/pogovarit'/) (t14) en lugar de поговорить (/pogovorit') 'hablar'.

(17) *nутишествовать (/putišestvovat'/)(t13) en vezde nутешествовать (/putešestvovat'/).

(18) наслаждаться *временим (/vremenim/) (t22) en vez de наслаждаться временем (/ vremenem/) 'disfrutar del tiempo [libre]'.

(19) в $^{*}$ кровотах /v krovotah/ (t14) en lugar de в кроватях /v krovatâh/ 'en las camas'.

\subsubsection{Errores con el signo blando}

Los errores en el uso del signo blando constituyen un 19,2\% del total, que corresponde a 24 errores. El signo blando cumple varias funciones en ruso. Entre ellas, indica que la consonante anterior está palatalizada. También sirve en algunos casos como signo de separación entre una consonante y las vocales $e[\mathrm{e}], \ddot{e}[\mathrm{\Theta}], u[\mathrm{i}], ю[\mathrm{u}]$, у $я$ [æ]. El análisis de errores reflejó casos de errores de sobreutilización del signo blando. En los ejemplos (20), (21) y (22) los aprendices añaden el signo blando a la letra л [1] cuando en realidad no es necesario. Este resultado nos indica que los aprendices tienen dificultad para distinguir la $\pi$ [1] dura y la $\pi\left[1^{\mathrm{j}}\right]$ palatalizada incluso en el nivel avanzado. Del mismo modo, se observaron errores de omisión del signo blando, por ejemplo, en (23) y (24).

(20) $м и$ *дольжни /dol'žni/ (t5) en lugar de мы должны /dolžny/ 'nosotros debemos'.

(21) *польный /pol'nyj/ дурак (t11) en lugar de полный/polnyj/ дурак 'un idiota total'.

(22) *больтаем /bol'taem/ (t22) en vez de болтаем/boltaem/ 'charlamos'.

(23) *уровен /uroven/ (t12) en vez de уровень /uroven'/ 'nivel'.

(24) *денги /dengi/ (t9) en vez de деньги /den'gi/ 'dinero'.

(25) * nюm /pût/ (t2) en vez de nьюm /p'ût/ 'beben'.

(26) используется *судями /sudâmi/ (t14) en lugar de используется судьями /sud'âmi/ 'se utiliza por los jueces'.

\subsection{Errores de omisión e inserción}

Los errores de omisión constituyen un 16\% del total (30 de 187). Algunos de estos errores están relacionados con el hecho de que las consonantes en algunas ocasiones no se pronuncian, por ejemplo, en (27) y (28). Otros errores de omisión parecen ser errores puntuales sin ningún tipo de interferencia de la L1 de los aprendices (ejemplos 29 y 30).

(27) казалось * страным /stranym/ (t13) en vez de казалось странным /strannym/ 'parecía raro'.

(28) * када (/kada/) (t9) en lugar de когда (/kogda/) 'cuándo'.

(29) *болье /bol'e/ (t3) en lugar de больше /bol'še/ 'más grande'.

(30) *удобтва /udobtva/ (t19) en vez de удобства /udobstva/ 'comodidades'.

Respecto a los errores de inserción, estos suponen un 7\% del total (13 de 187), siendo así los errores minoritarios que cometen los aprendices del nivel B2. Algunos se explican por 
las limitaciones del dominio del sistema fonológico de la L2, en concreto, la letra $九$ [æ] provoca dificultades, por lo que, a la hora de pronunciar o escribir esta letra los aprendices añaden una [i] por comodidad (véase ejemplo 31). Asimismo, en los ejemplos (32) y (33) se observan casos de sobregeneralización de la regla de duplicación de las consonantes, por ejemplo, Россия /rossija/ 'Rusia', сделанныцй/sdelannyj/ 'hecho'.

(31) *месияи /mesiâac/ (t5) en vez de месяй /mesâc/ 'mes'.

(32) *исчезновенния /isčeznovenniâ/ (t15) en vez de исчезновения/isčeznoveniâ/ 'desapariciones'.

(33) что *кассается /kassaetsja/ (t12) en lugar de что касается /kasaetsja/ 'en lo que se refiere'.

\subsection{Otros errores}

El apartado de otros errores constituye un 10\% de los errores en total (18 de 187). Dentro de este grupo se encuentran el uso de letras latinas ( 5 errores) y los casos de errores poco habituales (14 errores). Así, por ejemplo, en (34) y (35) el aprendiz sustituye la letra cirílica л [1] por la latina 1. Los casos de errores poco habituales incluyen, por ejemplo, la escritura de un símbolo no cirílico ni tampoco latino (véase ejemplo 36), el intercambio entre las letras $\kappa[\mathrm{k}]$ у $л[1]$ (ejemplo 37) y el intercambio de la $y$ [ซ] y la $b l$ [i] (ejemplo 38).

(34) *официаlьный (t22) en vez de официальный /oficial'nyj/ 'oficial'

(35) *бolbme (t23) en vez de больще /bol’še/ 'más grande'.

(36) *удочRa (t3) en vez de удочка /udočka/ 'caña de pescar'.

(37) *хаковаться /hakovat'sâ/ (t10) en vez de жаловаться/žalovat'sâ' 'quejarse'.

(38) *рубалки /rubalki/ (t14) en vez de рыбалки /rybalki/ 'pesca'.

\section{DISCUSIÓN Y CONLUSIONES}

Este trabajo pretendía alcanzar varios objetivos. En primer lugar, se planteaba analizar un corpus de producciones escritas de aprendices hispanohablantes de ruso como LE de nivel B2. En segundo lugar, se pretendía comparar los errores ortográficos en los corpus de producciones escritas de aprendices de dos niveles - A2 y B2.

Así, el análisis ha reflejado que los errores más frecuentes son los de sustitución. En concreto, el intercambio de las letras $\sigma-6[\mathrm{~b}]-[\mathrm{v}]$ y $u$ - $b l$ [i]-[i] prevalece en el corpus de aprendices de nivel B2. Ambos casos de sustitución grafémica están relacionados con las representaciones fonológicas de estas letras. En ruso, las letras $\sigma$ у в tienen dos representaciones fonológicas distintas ([b] y $[\mathrm{v}]$ o $\left.\left[\mathrm{b}^{\mathrm{j}}\right] \mathrm{y}\left[\mathrm{v}^{\mathrm{j}}\right]\right)$, mientras que en español las letras b y v se pronuncian de la misma manera $([\mathrm{b}] \mathrm{o}[\beta])$. En consecuencia, los aprendices hispanohablantes transfieren este aspecto fonológico de su L1 a la LE que están aprendiendo. En caso de sustitución de las letras $u$ - $b l$ [i]-[i], los errores observados se deben a que los aprendices hispanohablantes no distinguen la vocal cerrada central no redondeada [i] , por lo que la sobregeneralizan al sonido [i] y su respectiva representación grafémica $u$ [i]. Del mismo modo, los errores de sustitución relacionados con el uso del signo blando también son muy frecuentes. Especialmente, la dificultad se observa en el caso de palatalización innecesaria

3 En este caso el aprendiz no escribió exactamente la letra latina R sino un símbolo parecido a esta. 
de la letra $\pi$ [1]. Esto parece deberse a que en español no hay distinción entre los sonidos palatalizados y no palatalizados.

Los resultados coinciden con el análisis del corpus de aprendices de nivel A2 en el que se hallaron errores semejantes (Ogneva 2018) y con otros estudios que se centraron en el mismo aspecto (Klimova et al. 2017; Ohalina 2018). En la misma línea con Golikov (2008), en el corpus de trabajos de aprendices de nivel B2 se han identificado errores que tienen que ver con la similitud de escritura de las letras $u-u$ [s]-[6:] y $\varkappa_{-}-x[\mathrm{z}]-[\mathrm{x}]$. Además de los errores provocados por la L1 de los aprendices, se han identificado dificultades relacionadas con el sistema fonológico propio de la LE. En concreto, los alumnos de ruso como LE tienen problemas con los sonidos que se ven afectados por el proceso de reducción vocálica (o- $a[\mathrm{o}]-[\mathrm{a}]$ y $e-u$ [e]-[i]).

En cuanto al segundo objetivo, que consistía en comparar el número total de errores entre dos corpus de aprendices, no se han hallado diferencias significativas entre estos. Es decir, los aprendices no han avanzado significativamente en términos de escritura en ruso. No obstante, se observa que el número de errores disminuye conforme los alumnos adquieren mayores conocimientos. Así, al contrario de Ogneva (2018), en el corpus de aprendices B2 apenas se han identificado errores de intercambio de las letras $u-y / \mathrm{i}-\mathrm{u} /$. Esto indica que existe un cierto progreso en el proceso de aprendizaje del sistema ortográfico en ruso como LE, en concreto, se ha mejorado el nivel de la conciencia ortográfica de los aprendices. En futuras investigaciones conviene indagar en las razones de por qué algunas dificultades persisten incluso en los niveles avanzados.

En definitiva, a pesar de que el ruso es una lengua bastante alejada del español en términos de escritura, los aprendices hispanohablantes, cuyos trabajos forman parte del corpus analizado aquí, parecen ser capaces de emplearla con cierto nivel de corrección, estando aún afectados por su L1. Es necesario señalar algunas limitaciones del presente trabajo. Por ejemplo, contamos con un número relativamente pequeño de la muestra que forma parte del corpus. En futuras investigaciones se plantea ampliar este numero incluyendo trabajos de producción escrita de niveles más altos $(\mathrm{C} 1$ y $\mathrm{C} 2)$. De manera semejante, en futuros estudios habría que tener en cuenta la heterogeneidad de los alumnos matriculados en la EOI. No obstante, consideramos que los resultados aquí descritos pueden ayudar a desarrollar unidades didácticas centradas en los sonidos que presenten mayores dificultades con el objetivo de trabajar la conciencia fonológica de aprendices de ruso como LE y, consecuentemente, evitar posibles errores ortográficos.

\section{BIBLIOGRAFÍA}

Bondareva, V.V. y Loginova I.M. (2014): "Zvukovaja interferencija nositelej nekotoryh romanskih jazykov v oblasti russkogo vocalizma" [La transferencia sonora en el ámbito de vocalismo ruso por parte de algunos nativos de lenguas romance], Vestnik RUDN, 3, pp. 82-86.

Cook, V. (1997). "L2 users and English spelling". Journal of Multilingual and Multicultural Development, 18 (6), pp. 474-489.

Corder, S.P. (1967). "The significance of Learners Errors", INRAL, 5, pp. 161-170.

Corder, S. (1975). "Error Analysis, Interlanguage and Second Language Acquisition", Language Teaching \& Linguistics: Abstracts, 8 (4), pp. 201-218. doi:10.1017/S0261444800002822

Corder, S.P. (1981). Error Analysis and Interlanguage. Oxford: Oxford University Press.

Denes, G. (2011). Talking Heads: The neuroscience of language. Nueva York: Psychology Press. Taylor and Francis Group 
Denissenko, A. (2016). L'adquisició del rus com llengua estrangera en un context d'instrucció formal a l'aula: Complexitat, Correcció i Fluidesa i ús del cas en la producción escrita. Tesis doctoral, Universitat Pompeu Fabra, España. [Dispobible en https://www.tdx.cat/handle/10803/349216].

Fashola, O., Drum, P., Mayer, R. y Kang, J.S. (1996). "A cognitive theory of orthographic transitioning: Predictable errors in how Spanish-speaking children spell English words". American Educational Research Journal, 33, pp. 825-843.

Fernández, S. (1997). Interlengua y análisis de errores en el aprendizaje del español como lengua extranjera. Madrid: Edelsa.

Fries, Ch. (1945). Teaching and learning English as a Foreign Language. Ann Arbor: University of Michigan Press.

Golikov, S. (2008). Linguodidakticheskiye osnovy pedagogicheskogo proyektsirovaniya sistemy obucheniya ispantsev russkomu yaziku i testirovaniya. Tesis doctoral, Universidad Rusa de la Amistad de los Pueblos, Rusia.

Guzmán Tirado, R. (2007). Sobre la categoría de la taxis y su aplicación a la gramática comparada del ruso y del español. En Barros García, P., Aguila Escobar, G., Montoro del Arco, E.T. (eds.). Estudios lingüisticos, literarios e históricos. Homenaje a Juan Martínez Marín. Granada: Universidad de Granada, pp. 249-261.

Guzmán Tirado, R. y Herrador del Pino, M. (2000). Investigaciones de gramática funcional: la aspectualidad en ruso y en español. Granada: Universidad de Granada.

Guzmán Tirado, R. y Quero Gervilla, E.F. (2003). Tipología de la oración subordinada en ruso y en español. Madrid: Dykinson.

Hualde, J.I. (2005). The sounds of Spanish with audio CD. Cambridge University Press.

Ibrahim, M. (1978). "Patterns in spelling errors". English Language Teaching Journal, 32, pp. 207212.

International Phonetic Association (1999). Handbook of the International Phonetic Association: A guide to the use of the International Phonetic Alphabet. Cambridge University Press.

Jiménez González, J.E. y Ortiz González, M.R. (1995). Conciencia fonológica y aprendizaje de lectura: teoría, evaluación e intervención. Madrid: Editorial Síntesis.

Kirk, C. y Gillon, G.T. (2009). "Integrated Morphological Awareness Intervention as a Tool for Improving Literacy”. Language, Speech and Hearing Services in Schools, 40, pp. 341-351.

Klimova, Y. A., Yurchenko, N.V., Cherkashina Olga M. y Kulik S.S. (2017). "Sopostavitel'nyj analiz fonologicčeskih sistema russkogo i ispanskogo jazykov (v celja obučenija ispanogovorjasshih studentov russkomu proiznosheniju" [Análisis comparative de los sistemas fonologicos de español y ruso (con el objetivo de enseñar la pronunciacion rusa a los aprendices hispanohablantes)], Kazanskij pedagogicheskij zhurnal, 6, pp. 108-110.

Lado, R. (1957). Linguistics Across Cultures. Applied Linguistics for Language Teachers. Ann Arbor: University of Michigan Press.

Martínez-Celdrán, E., Fernández-Planas, A. M. y Carrera-Sabaté, J. (2003). Illustrations of the IPA. Castilian Spanish. Journal of the International Phonetic Association, 33 (2), pp. 255-259.

[MCER] Consejo de Europa (2002). Marco común europeo de referencia para las lenguas: aprendizaje, enseñanza, evaluación. Madrid: Instituto Cervantes, Ministerio de Educación, Cultura y Deporte, Anaya. [Disponible en: http://cvc.cervantes.es/ensenanza/biblioteca_ele/marco/cvc_mer.pdf].

Míguez Álvarez, C.M. (2018). "Influencia de las conciencias fonológica y morfológica en la adquisición de la lectura”. Estudios interlingüísticos, 6, pp. 96-115.

Muñoz-Basols, J. y Bailini, S. (2018). "Análisis y corrección de errores”. En Muñoz-Basols, J., Gironzetti, E., y Lacorte, M. (eds.). The Routledge handbook of Spanish language teaching (pp. 94-108). New York: Routledge.

Ogneva, A. (2018). "Spelling errors in L2 Russian: evidence from Spanish-speaking students". Estudios interlingüísticos, 6, pp. 116-131. 
Ogneva, A. (2019). "Análisis de errores e interferencias interlingüísticas en ruso como LE por aprendices hispanohablantes". RAEL: revista electrónica de lingüística aplicada, 18 (1), pp. 56-71.

Ohalina, A.A. (2018). "Tipičnyje akcentologičeskije trudnosti isponogovorjaŝih studentov pri izučenii russkogo jazyka" [Las dificultades típicas de los aprendices hispanohablantes en el aprendizaje de ruso], en Pogorelova, S.D. (ed.). Problemy inzhenernogo i social'no-economicheskogo obrazovania $v$ tehnicheskom vuze v uslovijah modernizacii vysshego obrazovania. Materialy IX mezhdunarodnoj nauchno-metodicheskoj konferencii. Tjumen': Tjumenskij industrial'nyj universitet.

Park, C. (2011). The influence of L1 phonological and orthographic system in L2 spelling: a comparison of Korean learners of English and native speaking children. Tesis doctoral, Bull State University, EE.UU. [Disponible en https://cardinalscholar.bsu.edu/bitstream/handle/123456789/194900/ ParkC_2011-3_BODY.pdf? sequence=1].

Popova, G.G. (2007). Modal'nye glagoly "moch" $i$ "xotet" v russkom $i$ "poder" $i$ "querer" $v$ ispanskom yazikax [Los verbos modales "moch" y "xotet” en ruso y "poder" y "querer" en español]. Tesis doctoral, Rostovskiy Gosudarstvenniy Pedagogicheskiy Universitet, Rusia.

Quero Gervilla, Á. (2004). “Análisis de errores e interlengua en la adquisición de las preposiciones en ruso por hispanohablantes", Cuadernos de Rusística Española, 1, pp. 89-104.

Quero Gervilla, Á. (2005). Análisis de errores en la adquisición del caso en ruso por hispanohablantes. Madrid: Dykinson.

Sánchez Puig, M., Drozdov Díez, T., Pérez Molero, P., Stasyuk, V. y Usiatínskaya, I. (2005). Curso completo de la lengua rusa. Nivel básico, intermedio y avanzado. Madrid: Ediciones Hispano Eslavas.

Selinker, L. (1972). "Interlanguage”, IRAL-International Review of Applied Linguistics in Language Teaching, 10 (1-4), pp. 209-232.

Selinker, L. (2013). Rediscovering interlanguage. New York: Routledge.

Selinker, L. (2014). "Interlanguage 40 years on: Three themes from here". En Han, Z. y Tarone, E. (eds.), Interlanguage: Forty years later. Amsterdam: John Benjamins Publishing Company, pp. 221-246.

Sun-Alperin, M. K. y Wang, M. (2008). "Spanish-speaking children's spelling errors with English vowel sounds that are represented by different graphemes in English and Spanish words". Contemporary Educational Psychology, 33 (4), pp. 932-948.

Vinogradov, V. y Miloslavskiy, I. (1979a). "Russkaya gramatica v sopostavlenii s ispanskoy: sxodstva i razlichiya. Statya pervaya. Rod i odyshivlennost"”, Russkiy yazik za rubezhom, 2 (58), pp. 69-70.

Vinogradov, V. y Miloslavskiy, I. (1979b). "Russkaya gramatica v sopostavlenii s ispanskoy: sxodstva i razlichiya. Statya vtoraya. Chislo russkix i ispanskix suschestvitelnyx", Russkiy yazik za rubezhom, 5 (61), pp. 69-72.

Vinogradov, V. y Miloslavskiy, I. (1980). "Russkaya gramatica v sopostavlenii s ispanskoy: sxodstva i razlichiya. Statya tret'ya. Russkiy i ispanskiy glagol”, Russkiy yazik za rubezhom, 4 (66), pp. 48-53.

Vinogradov, V. y Miloslavskiy, I. (1986). Sopostavitel'naya morfología russkogo i ispansogo yazykov. Moscú: Moskva. 